\title{
COMMUNICATION
}

\section{Découverte d'un lymphome $B$ non hodgkinien sur une lésion ostéolytique mandibulaire associée à une paresthésie labio -mentonnière.}

\author{
Fénelon M1', Bercault B, Marteau JM, Lauverjat Y, Catros S, Calmettes C, Fricain JC2 \\ 1. Service d'Odontologie et de Santé Buccale, Hôpital Pellegrin - Bordeaux \\ 2. Bioingénierie Tissulaire (BIOTIS) Inserm : U1026, Université Victor Segalen - Bordeaux II
}

\section{Introduction}

La survenue de paresthésie labio-mentonnière associée à une lésion ostéolytique mandibulaire peut évoquer le diagnostic d'ostéite ou faire suspecter la présence d'une lésion maligne. Les lymphomes non hodgkinien $(\mathrm{LNH})$ extra ganglionnaires touchent essentiellement le tube digestif et les voies aéro-digestives supérieures. De rares cas d'atteinte extra-ganglionnaire localisée à la mandibule ont été rapportés (Dinakar 2010 ; Okahata 2014).

\section{Observation}

Un patient de 64 ans, sans antécédent médicochirurgicaux particuliers, a consulté dans le service d'Odontologie pour des douleurs dentaires évoluant depuis plusieurs mois. L'interrogatoire mettait en évidence des douleurs à type de décharge électrique évoluant par intermittence. Une paresthésie labiomentionnière était retrouvée à l'examen clinique exobuccal. II n'existait pas d'asymétrie faciale ni de limitation de l'ouverture buccale et on ne retrouvait pas d'adénopathie. L'examen clinique endobuccal mettait en évidence une discrète tuméfaction mandibulaire droite non douloureuse à la palpation. L'examen tomodensitométrique objectivait une lésion mandibulaire ostéolytique, mal délimitée, allant de l'apex de la dent 47 et dépassant la 48 qui était incluse. Les corticales étaient respectées. II était difficile d'individualiser le nerf alvéolaire inférieur du reste de la lésion. L'avulsion de la dent de sagesse associée à un prélèvement de la lésion ont été réalisés sous anesthésie locale. L'examen anatomopathologique mettait en évidence un lymphome malin non hodgkinien de phénotype B à grandes cellules. Le patient a été confié au service d'hématologie où un bilan par Scanner puis TEP-Scan a été réalisé ne montrant pas d'extension de la maladie. II s'agissait d'un LNH de stade IE (site extra ganglionnaire). Le patient a eu 4 cures d'immunochimiothérapie qui seront éventuellement associées à une radiothérapie après réévaluation par TEP-Scan. Après 3 cures, on observait une diminution des douleurs ainsi que de la paresthésie labio-mentonnière.

\section{Discussion / Conclusion}

Le LNH de localisation mandibulaire se manifeste le plus souvent par : une tuméfaction mandibulaire, des douleurs, des troubles neurologiques et/ou des mobilités dentaires. Les diagnostics différenciels fréquemment évoqués sont une ostéite, pouvant être responsable d'un retard diagnostique, ainsi qu'un sarcome ou un carcinome pouvant amener à des chirurgies inutilement invasives (Barchaud 1992, Wrong 2013). En effet, après avoir établi le diagnostic de certitude, qui repose sur une analyse histopathologique, le traitement nécessite une chimiothérapie et/ou une radiothérapie sans chirurgie associée.

mathildefenelon@live.fr

(C) The authors, published by EDP Sciences. This is an Open Access article distributed under the terms of the Creative Commons Attribution License 4.0 (http://creativecommons.org/licenses/by/4.0/). 